\title{
Weather Based Agro-Met Advisory to Enhance the Production and Income of the Farmers under Changing Climate Scenario of Central Plain Zone of Uttar Pradesh, India
}

\author{
Nauashad Khan, Ajay Kumar*, C.B. Singh, Vijay Dubey and Nitish Kumar \\ Department of Agronomy, CSA University of Agriculture and Technology, \\ Kanpur - 208 002, India \\ *Corresponding author
}

K e y w o r d s
Agro-Met
$\begin{aligned} & \text { Advisory, Enhance, } \\ & \text { Climate, Central } \\ & \text { plain zone }\end{aligned}$
Article Info
$\begin{aligned} & \text { Accepted: } \\ & \text { 04 October } 2018 \\ & \text { Available Online: } \\ & 10 \text { November } 2018\end{aligned}$

\section{Introduction}

Weather is one of the most important factors determining success or failure of agricultural production. It affects on every phase of plant growth and development. Any variability in the weather during the crop season, such as delay in the monsoon, excessive rains, flood, droughts, spells of too-high or too-low temperatures would affect the crop growth and
Agricultural production depends upon many factors, of which weather is the major factor. Weather varies with space and time; hence, its forecast can help to minimize the farm losses through proper management of agricultural operations. The complete avoidance of all farm losses due to weather factor is not possible but it can be minimized to some extent by making adjustments through timely and accurate information of weather forecast. Weather forecast and weather based agro-met advisories help in increasing the economic benefit to the farmers by suggesting them the suitable management practices according to the weather conditions. A study was, therefore, undertaken on adaptation of agro-met advisory bulletin and economic impact of agro-met advisory services for maize and rice during Kharif 2015. For assessing the impacts of agro-met advisory services, users of agromet advisory services (AAS) and non-users of agro-met advisory services (non AAS) were selected from adopted NICRA villages. Results showed that the farmers, who followed the agro-met advisories, are able to reduce the input cost and increases net profit as compared to the non AAS farmers in Maize and rice. This might be resulted due to the crop management done by the farmers as per to agro-met advisory bulletins. Thus, the application of agro-met advisory bulletin, based on current and forecast weather is a useful tool for enhancing the production and income of farmers. 
on the various day-to-day farming operations i. e. sowing, weeding, time of pesticides spray, irrigation scheduling, fertilizer application etc. and overall crop management. Weather forecast helps to increase agriculture production, reduce losses, risks, reduce costs of inputs, improve quality of yield, increase efficiency in the use of water, labor and energy and reduce pollution with judicious use of agricultural chemicals. Rathore et al., (2001) discussed the weather forecasting scheme operational at National Centre for Medium Range Weather Forecast for issuing location specific weather forecast five days in advance. Damrath et al., (2001) reported that the statistical interpretation methods are used to increase the reliability of the precipitation forecast. The benefit by the farmers using agro-met advisory bulletin and weather forecast for making farm-level decisions by farmers from different village have been discussed in this paper.

\section{Materials and Methods}

The Integrated Agro-met Advisory Services located in the Department of Agronomy (Agrometeorology), Chandra Shekhar Azad University of Agriculture \& Technology, Kanpur (U.P.) has been serving the farming community. Progressive farmers have been taking keen interest in the agro-advisories and are the foremost beneficiaries. The major objective of this programme is to advise timely and need-based crop management practices. Weather forecast on rainfall, maximum and minimum temperature, wind speed, wind direction, cloud cover, maximum and minimum humidity are being received on every Tuesday and Friday from IMD, New Delhi. Once the forecast was received, the experts' opinion from different disciplines was obtained. Based on the advice, the agro advisories are being prepared on every Tuesday and Friday in Hindi as well as in English. Bulletins are regularly communicated to the farmers on real time basis through telephone/ E-mail/SMS. Agro-met advisory bulletins are also sent by E-mail to local Hindi newspapers for publication in both Hindi and English language. The bulletins are also sent to KVK Daleep Nagar, NICRA Village of Daleep Nagar, Block-Shivrajpur (Kanpur Dehat) and Village-Saibasu Block-Bilhur (Kanpur Nagar), e-choupal, Krishi Darsan and All India Radio through E-mail messages.

The weather forecast based agro-advisory bulletin contains a summary of previous weeks' weather, deviation of weather from the normal value, weather forecast information for the next five days, crop management, which is based on weather forecast and giving warning to the farmers well in advance, regarding rainfall variation, its amount and other weather variables including pest/disease problems. Thus, farmers can decide on crop management options, application of nutrients and strategies to overcome other problems.

Weather based agro-met advisory to enhance the production and income

Weather forecast and weather based agro-met advisories help in increasing the economic benefit to the farmers by suggesting them the suitable management practices as per the prevailing weather conditions. A study was, therefore, undertaken on adaptation of agromet advisory bulletin and economic impact of agro-met advisory services for rice and maize during Kharif 2015.

For assessing the impacts of agro-met advisory services, users of agro-met advisory services (AAS) and non-users of agro-met advisory services (non AAS) were selected for wheat and rice crop. The study area lays around $35 \mathrm{~km}$ of village Daleep Nagar, (Kanpur Dehat) and Village-Saibasu BlockBilhur (Kanpur Nagar), are $35 \mathrm{Km}$ and $54 \mathrm{~km}$ away from Kanpur, respectively. 
Fig.1 Economic impact of AAS and Non AAS on Rice (Rs./ha.) in Daleep Nagar

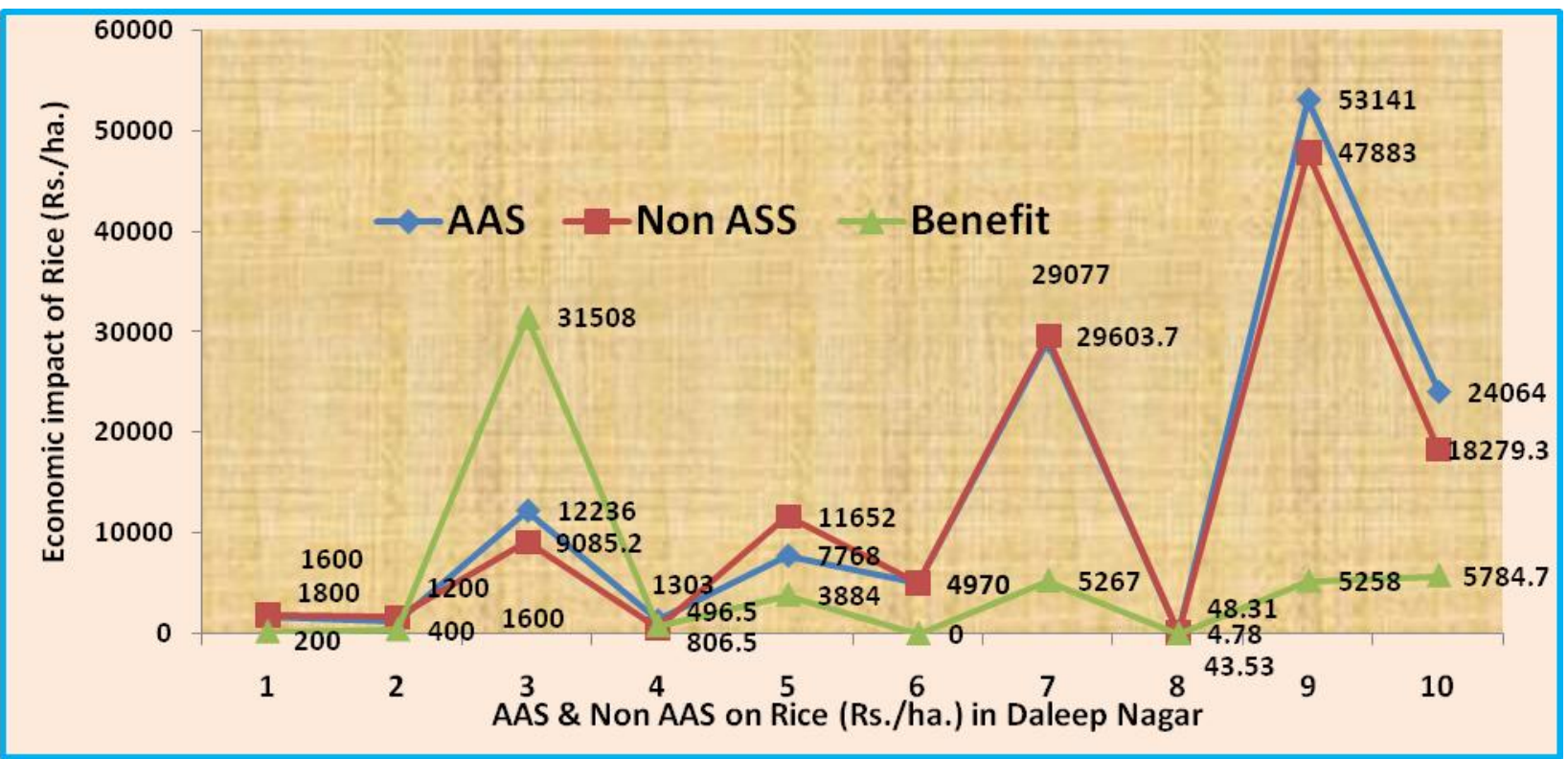

Fig.2 Economic impact of AAS and Non AAS on Maize (Rs./ha.) in Saibasu

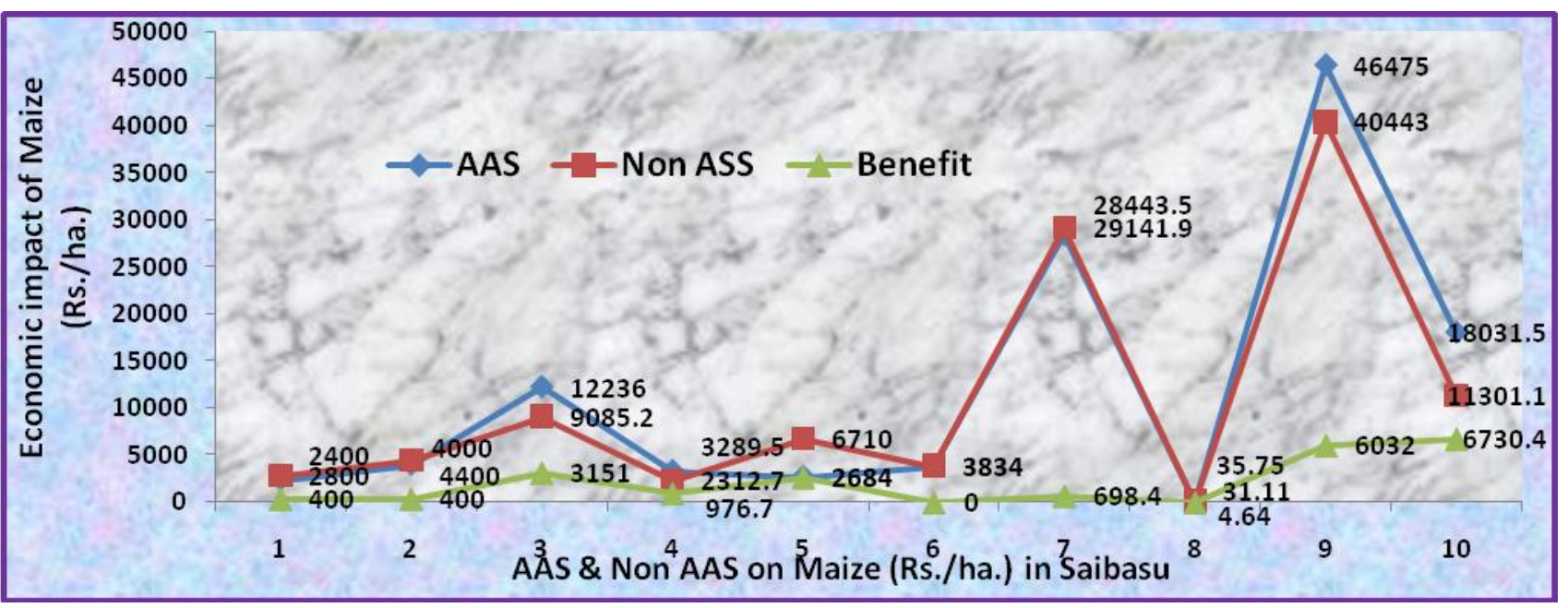

Table.1 Economic impact of AAS and Non AAS farmers on Rice (Rs./ha.) in Daleep Nagar during Kharif 2015-16

\begin{tabular}{|c|c|c|c|c|c|c|c|c|c|c|}
\hline Type & $\begin{array}{c}\text { Preparation/ } \\
\text { Sowing }\end{array}$ & Seed & $\begin{array}{c}\text { Fertilizer } \\
\& \\
\text { Manure }\end{array}$ & $\begin{array}{l}\text { Pesticides/ } \\
\text { Insecticide/ } \\
\text { Herbicide }\end{array}$ & Irrigation & $\begin{array}{l}\text { Harvesting/ } \\
\text { Threshing }\end{array}$ & Input & $\begin{array}{l}\text { Yield } \\
\text { (q/ha.) }\end{array}$ & Rs. & Net profit \\
\hline AAS & 1600.0 & 1200.0 & 12236.0 & 1303.0 & 7768.0 & 4970.0 & 29077.0 & 48.31 & 53141.0 & 24064.0 \\
\hline Non ASS & 1800.0 & 1600.0 & 9085.2 & 496.5 & 11652.0 & 4970.0 & 29603.7 & 43.53 & 47883.0 & 18279.3 \\
\hline Benefit & 200.0 & 400.0 & 31508.0 & 806.5 & 3884 & 0.0 & 526.7 & 4.78 & 5258.0 & 5784.7 \\
\hline
\end{tabular}


Table.2 Economic impact of AAS and Non AAS farmers on Maize (Rs./ha.) in Saibasu during Kharif 2015-16

\begin{tabular}{|l|c|c|c|c|c|c|c|c|c|c|}
\hline Type & $\begin{array}{c}\text { Preparation/ } \\
\text { Sowing }\end{array}$ & Seed & $\begin{array}{c}\text { Fertilizer } \\
\& \\
\text { Manure }\end{array}$ & $\begin{array}{l}\text { Pesticides/ } \\
\text { Insecticide/ } \\
\text { Herbicide }\end{array}$ & Irrigation & $\begin{array}{l}\text { Harvesting/ } \\
\text { Threshing }\end{array}$ & Input & $\begin{array}{l}\text { Yield } \\
(\mathbf{q} / \text { ha.) }\end{array}$ & Rs. \\
\hline AAS & 2400.0 & 4000.0 & 12236.0 & 3289.5 & 2684.0 & 3834.0 & 28443.5 & 35.75 & 46475.0 & 18031.5 \\
\hline Non ASS & 2800.0 & 4400.0 & 9085.2 & 2312.7 & 6710.0 & 3834.0 & 29141.9 & 31.11 & 40443.0 & 11301.1 \\
\hline Benefit & 400.0 & 400.0 & 3151.0 & 976.7 & 2684.0 & 0.0 & 698.4 & 4.64 & 6032.0 & 6730.4 \\
\hline
\end{tabular}

\section{Results and Discussion}

Results showed that the farmers who followed the agro-met advisories are able to reduce the input cost up to $1.8 \%$ in rice, $2.4 \%$ in maize and increases the net profit by $11 \%$ in rice (Table 1) and net profit by $14 \%$ in maize (Table 2), respectively, as compared to the non AAS farmers, who did not follow the weather based information. AAS farmers were able to reduce the input cost up to Rs. 526.7/ha in rice and Rs. 698/ha in maize. Increases in the net profit were Rs. 5785/ha in rice and Rs. 6730/ha in maize compared to the non AAS farmers. More net returns of AAS farmers over non-AAS farmers can be due to low input cost, following weather based management practices and timely management of pests and diseases. This profit was due to the crop management done by the farmers such as timely land preparation and sowing, adoption of recommended seed rate and suitable varieties, timely weeding, harvesting and irrigation and pesticide applications, suggested through agro-met advisory bulletins (Fig. 1 and 2).

The studies showed that the application of agro-met advisory bulletin, based on current and forecasted weather is a useful tool for enhancing the production and income. AAS farmers received weather forecast based agroadvisories, including optimum use of inputs for different farm operations. Due to judicious and timely utilization of inputs, production cost for the AAS farmers reduced. The increased yield level and reduced cost of cultivation led to increased net returns.

\section{References}

Damrath, U., Doms, G., Friihwald, D., Heise, E., Richter, B. and Steppeler, J. (2000). Operational quantitative precipitation forecasting at the German Weather Service. J of Hydrology. 239: 260-285.

Rathore, L. S., Gupta, Akhilesh and Singh, K. K. (2001). Medium range weather forecasting and agricultural production. Journal of Agric. Physics. 1(1): 43

Vashisth Ananta, Singh, R., Das, D.K. and Baloda, R. (2013). Weather Based Agromet Advisories for Enhancing the Production and Income of the Farmers under Changing Climate Scenario. International Journal of Agriculture and Food Science Technology. ISSN 22493050, Volume 4, Number 9 (2013), pp. 847-850.

\section{How to cite this article:}

Nauashad Khan, Ajay Kumar, C.B. Singh, Vijay Dubey and Nitish Kumar. 2018. Weather Based Agro-Met Advisory to Enhance the Production and Income of the Farmers under Changing Climate Scenario of Central Plain Zone of Uttar Pradesh, India. Int.J.Curr.Microbiol.App.Sci. 7(11): 221-224. doi: https://doi.org/10.20546/ijcmas.2018.711.027 